\title{
Effect of different reconstruction algorithms on coronary artery calcium scores using the reduced radiation dose protocol: a clinical and phantom study
}

\author{
Yu-Kun Pan ${ }^{1}$, Ming-Hua Sun ${ }^{2}$, Jia-Jia Wang ${ }^{1}$, Xing-Biao Chen ${ }^{3}$, Xiao-Jing Kan ${ }^{2}$, Ying-Hui Ge ${ }^{2}$, \\ Zhi-Ping Guo ${ }^{4}$ \\ ${ }^{1}$ Department of Radiology, Central China Fuwai Hospital, People's Hospital of Zhengzhou University, Zhengzhou, China; ${ }^{2}$ Department of \\ Radiology, Henan Provincial People's Hospital, Department of Radiology of Central China Fuwai Hospital, Central China Fuwai Hospital of \\ Zhengzhou University, Zhengzhou, China; ${ }^{3}$ Clinical Science, Philips Healthcare, Shanghai, China; ${ }^{4}$ Central China Fuwai Hospital, Zhengzhou, \\ China
}

Correspondence to: Ying-Hui Ge. Department of Radiology, Henan Provincial People's Hospital, Department of Radiology of Central China Fuwai Hospital, Central China Fuwai Hospital of Zhengzhou University, Zhengzhou 450003, China. Email: cjr.geyinghui@vip.163.com; Zhi-Ping Guo. Central China Fuwai Hospital, Zhengzhou 450003, China. Email: guozhiping74@hotmail.com.

Background: This study aimed to evaluate the effects of different iterative reconstruction (IR) algorithms on coronary artery calcium (CAC) score quantification using the reduced radiation dose (RRD) protocol in an anthropomorphic phantom and in patients.

Methods: A thorax phantom, containing 9 calcification inserts with varying hydroxyapatite (HA) densities, was scanned with the reference protocol $[120 \mathrm{kv}, 80 \mathrm{mAs}$, filtered back projection (FBP)] and RRD protocol (120 kV, 20-80 mAs, 5 mAs interval) using a 256-slice computed tomography (CT) scanner. Raw data were reconstructed with different reconstruction algorithms [iDose ${ }^{4}$ levels 1-7 and iterative model reconstruction (IMR) levels 1-3]. Signal-to-noise ratio (SNR), contrast-to-noise ratio (CNR), and Agatston score (AS) were calculated for each image series. The correction factor was derived from linear regression analysis between the reference image series and other image series with different parameters. Additionally, 40 patients were scanned with the RRD protocol $(50 \mathrm{mAs})$ and reconstructed with FBP, iDose ${ }^{4}$ level 4, and IMR level 2. AS was calculated for the 3 -group image series, and was corrected by applying a correction factor for the IMR group. The agreement of risk stratification with different reconstruction algorithms was also analyzed.

Results: For the phantom study, the iDose ${ }^{4}$ and IMR groups had significantly higher SNR and CNR than the FBP group (all $\mathrm{P}<0.05)$. There were no significant differences in the total AS after comparing image series reconstructed with iDose ${ }^{4}$ (level 1-7) and FBP (all P>0.05), while AS from the IMR (level 1-3) image series were lower than the FBP group (all $\mathrm{P}<0.05$ ). The tube current of $50 \mathrm{mAs}$ was determined for the clinical study, and the correction factor was 1.14. For the clinical study, the median AS from the iDose ${ }^{4}$ and IMR groups were both significantly lower compared to the FBP image series [(112.89 (63.01, 314.09), 113.22 $(64.78,364.95)$ vs. 118.59 (65.05, 374.48), both $\mathrm{P}<0.05]$. After applying the correction factor, the adjusted AS from the IMR group was not significantly different from that of the FBP group $[126.48(69.62,355.85)$ vs. 118.59 (65.05, 374.48), $\mathrm{P}=0.145]$. Moreover, the agreement in risk stratification between FBP and IMR improved from 0.81 to 0.85 .

Conclusions: The RRD CAC scoring scan using the IMR reconstruction algorithm is clinically feasible, and a correction factor can help reduce the AS underestimation effect.

Keywords: Multidetector computed tomography (multidetector CT); coronary artery disease (CAD); vascular calcification; image reconstruction 
Submitted Mar 14, 2020. Accepted for publication Dec 24, 2020.

doi: 10.21037 /qims-20-437

View this article at: http://dx.doi.org/10.21037/qims-20-437

\section{Introduction}

Coronary artery calcium (CAC) scoring is a reliable, reproducible, non-invasive imaging technique used to assess asymptomatic patients with intermediate cardiac risk. It is also a good predictor of future cardiovascular risk independent of conventional risk scores, such as the Framingham risk score (1-5). The Agatston score (AS) method, which was first introduced in 1990, is the main system for the quantification of CAC (6). Although the AS has been widely used to identify and stratify patients at risk for coronary artery disease (CAD), it has certain limitations (7). Its major drawback is that the weighting factor is strongly dependent on image noise since it is based on maximum computed tomography (CT) numbers. Also, AS increases nonlinearly with an increase in coronary calcium. The second limitation is that AS is calculated and analyzed from the images reconstructed with the filtered back projection (FBP) algorithm, which requires a high radiation dose.

The low tube voltage method is widely used to reduce radiation doses for CAC scans (8). Nevertheless, low tube voltage produces a higher reduction value for calcium lesions, which requires an adaptation of the threshold of $130 \mathrm{HU}$ configured by the AS method (9). Another limitation when using the low tube voltage approach is that it increases image noise and beam-hardening artifacts, which may impact the calcium lesion volume evaluation (9).

The iterative reconstruction (IR) algorithm is another approach that has been proposed to reduce image noise and lower the radiation dose for CAC scans $(10,11)$. Previous studies have shown that the hybrid IR (HIR) algorithm could reduce the radiation dose by $60.6 \%$ while maintaining equivalent image quality as the FBP algorithm (11). However, other studies $(10,12,13)$ have reported that AS is lower with HIR compared to that with FBP.

The iterative model reconstruction (IMR) algorithm was recently introduced among the latest generation of the IR algorithms, and is a model-based full IR algorithm (14). IMR reduces image noise, blooming artifacts, and measurement variability, thus producing stable and reproducible CAC scores. Although IMR can reduce the radiation dose by $80 \%$ without compromising image quality, some studies have shown that CAC scores from IMR are underestimated compared to those from $\operatorname{FBP}(15,16)$. Yet, the effects of IMR algorithms combined with different low tube currents on CAC scores have not been extensively discussed, and currently there is no method that can effectively offset the CAC score underestimation effect for risk stratification.

The purpose of this study was thus to evaluate the effects of different reconstruction algorithms on CAC scores using a reduced radiation dose (RRD) protocol in vitro and in vivo. Furthermore, we derived correction factors for CAC scores from IMR images, and applied this correction factor to clinical data so as to assess the effect on CAC scoring and risk stratification in comparison with FBP images.

\section{Methods}

\section{Pbantom}

An anthropomorphic cardio CT phantom comprising thorax and cardiac calcification inserts (QRM Cardio-Phantom; QRM GmbH) was used in this study (Figure 1) (17). The thorax phantom included artificial lung lobes, a spine insert, and the shell of soft tissue-equivalent materials. The cardiac calcification inserts contained 9 cylindrical calcifications with different sizes and hydroxyapatite (HA) densities of 200, 400, or $800 \mathrm{mg} / \mathrm{cm}^{3}$. It also contained 2 larger calibration inserts, with 1 made of water-equivalent material, while the other contained $200 \mathrm{mg} / \mathrm{cm}^{3} \mathrm{HA}$.

\section{Patient population}

The institutional review board of our institution approved this study, and all patients signed written informed consent. The approval number was No.9 of the Ethical Review (in 2019).

A total of 50 patients with suspected CAD who underwent CAC scans between July and September 2019 were enrolled in this study. Patients with severe arrhythmia $(n=1)$, previous coronary artery bypass grafting or coronary stenting $(n=4)$, prosthetic valve replacement $(n=2)$, pacemakers or implantable cardioverter defibrillators $(\mathrm{n}=1)$, or patients with no coronary calcifications $(\mathrm{n}=2)$ were excluded. Finally, 40 patients were included.

\section{Image acquisition and reconstruction}

Both the phantom and patients were scanned with a 


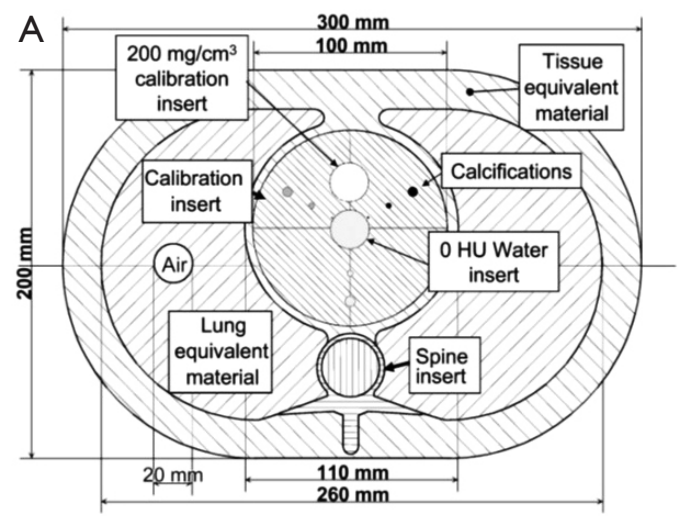

B

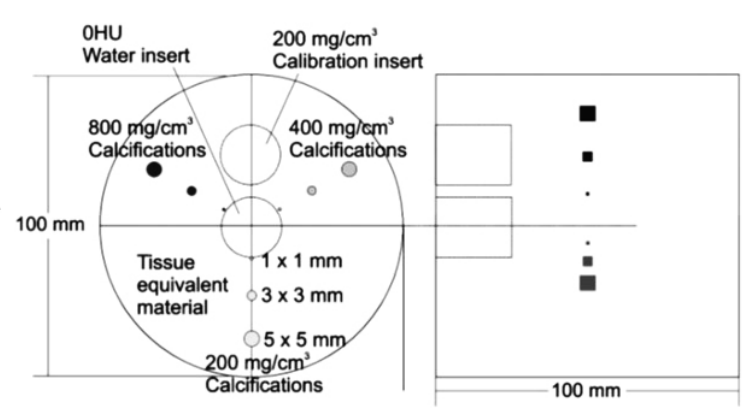

Figure 1 Characteristic of phantom. (A) Diagram of the frontal view of the anthropomorphic phantom body with the calibration insert. (B) Diagrams of the frontal (left) and side (right) views of the calibration insert, with 9 different calcifications and 2 large calibration inserts (0 HU water and $200 \mathrm{mg} / \mathrm{cm}^{3}$ calcium HA) (17).

256-slice CT scanner (Brilliance iCT, Philips Healthcare). The phantom was scanned with the prospective electrocardiographic (ECG)-triggered acquisition mode with a simulated heart rate of $65 \mathrm{bpm}$ (Model 430B Patient Simulator, Medi Cal Instruments Inc.). The data were acquired with a tube voltage of $120 \mathrm{kV}$, and different tube currents from 20 to $80 \mathrm{mAs}$ with an interval of $5 \mathrm{mAs}$. The phantom was scanned 3 times for each data acquisition parameter setting. All the acquisition data sets were reconstructed with the FBP algorithm, HIR algorithm (iDose ${ }^{4}$ level 1-7), and the full IR algorithm (IMR level 1-3).

Patients were scanned using a CAC scoring protocol with a tube voltage of $120 \mathrm{kV}$ and a tube current of $50 \mathrm{mAs}$ using the prospective ECG-triggered acquisition mode. All the acquisition data sets were reconstructed with the FBP algorithm, HIR algorithm (iDose ${ }^{4}$ level 4), and the full IR algorithm (IMR level 2). The other acquisition and reconstruction parameters were the same for both the phantom and patient scans and included a gantry rotation time of $0.25 \mathrm{~s}$, an adaptive detector collimation varying from 96 to 192 in steps of $8 \mathrm{~mm} \times 0.6 \mathrm{~mm}$, a reconstruction filter of cardiac standard (CB), an image slice thickness of $2.5 \mathrm{~mm}$, and an increment of $2.5 \mathrm{~mm}$.

\section{Calcium score analysis}

All the image series were reviewed and analyzed on a commercially available workstation (IntelliSpace Portal, version 7.0, Philips Healthcare). For the phantom study, a region of interest (ROI) with an area of $200 \mathrm{~mm}^{2}$ was drawn on the 2 calibration inserts (material of water equivalent and
$200 \mathrm{mg} / \mathrm{cm}^{3} \mathrm{HA}$ ) to measure mean CT value and standard deviation (SD). Furthermore, signal-to-noise (SNR) and contrast-to-noise ratios (CNR) were calculated using the following formulas:

$$
\begin{aligned}
& S N R=\frac{\text { CT Value }_{\text {HA-200 }}}{S D_{\text {Water-equivalent }}} \\
& C N R=\frac{C T \text { Value }_{H A-200}-\text { CTValue }}{\text { Water-equivalent }}
\end{aligned}
$$

One radiologist with 3 years of experience in cardiovascular diagnosis independently performed the CAC scoring for all image series using a commercially available software application (Heartbeat-CS, Philips Healthcare) according to the AS method (5). This application automatically marked the CAC plaques with an area greater than $1 \mathrm{~mm}^{2}$ and a CT value greater than $130 \mathrm{HU}$. Then, a calcium area was confirmed or modified manually, and an AS was automatically calculated. A senior radiologist with 12 years of experience in cardiovascular diagnosis independently confirmed all the CAC score results.

Patients were classified into the following 4 risk categories based on the AS values (18): 0, 1-100, 101-400, and $>400$.

\section{Correction factor}

In the phantom study, AS from the FBP image series with a tube current of $50 \mathrm{mAs}$ was set as the standard reference. Linear regression analysis for AS was performed between the reference image series and other image series 

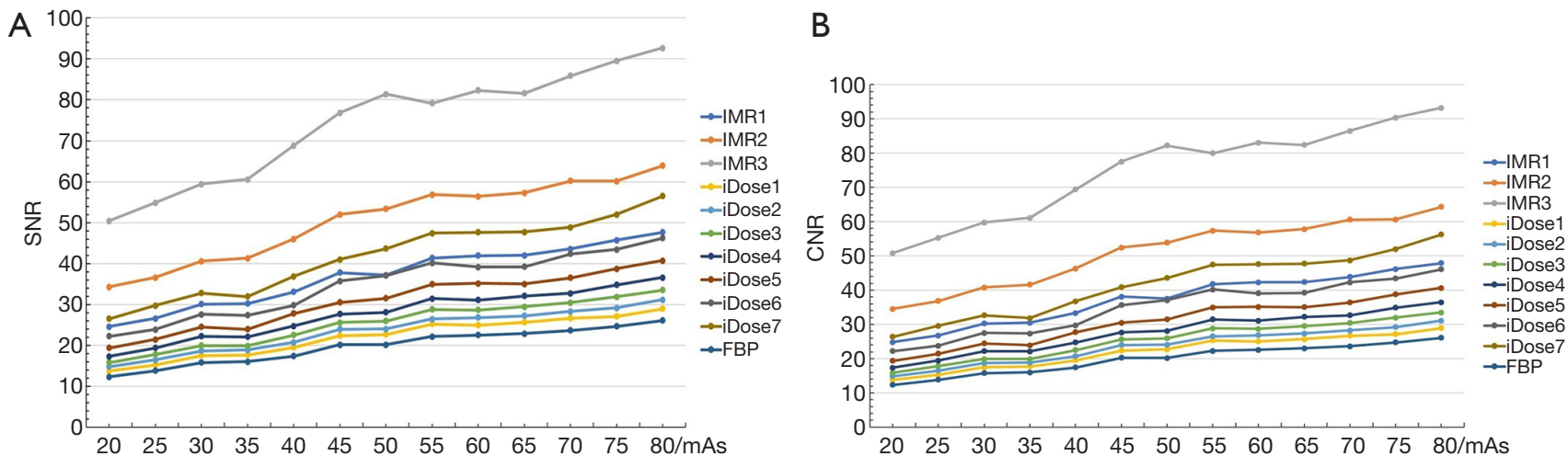

Figure 2 SNR (A) and CNR (B) of different reconstruction algorithms with different protocols. SNR, signal-to-noise ratio; CNR, contrastto-noise ratio; FBP, filtered back projection; IMR, iterative model reconstruction; IMR1, IMR level 1; IMR2, IMR level 2; IMR3, IMR level 3;

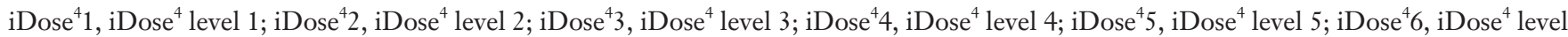
6; iDose ${ }^{4} 7$, iDose ${ }^{4}$ level 7.

with different parameters. The slope from the regression equations was used as the correction factor.

When analyzing patients, the obtained correction factor was applied to generate the corrected AS, and risk reclassification was performed.

\section{Radiation dose}

The volume CT dose index (CTDIvol, mGy), and the dose length product (DLP, $\mathrm{mGy} \cdot \mathrm{cm}$ ) were recorded for each scan. The effective dose $(\mathrm{ED}, \mathrm{mSv})$ was estimated by multiplying DLP and a conversion factor for chest examination $\left(k=0.014 m S v \times m G y^{-1} \times \mathrm{cm}^{-1}\right)(19)$.

\section{Statistical analysis}

For the phantom study, the AS of the image series using different reconstruction algorithms and radiation doses was compared to that of the reference image series using a paired $t$-test. Regression analysis was performed to assess the relationship of AS between the reference image series and others. The SNR and CNR were compared using an independent $t$-test.

For the clinical study, the AS of the 3 different reconstruction algorithms is presented as median and interquartile because the scores were not normally distributed. AS from iDose ${ }^{4}$ and the IMR image series were compared to the FBP image series using the Wilcoxon signed-ranks test. Agreement in risk stratification between FBP and IMR was evaluated by using Cohen's kappa ( $\kappa)$ statistic. Excellent agreement was defined as $\kappa>0.80$, good agreement was $0.61-$ 0.80 , moderate agreement was $0.41-0.60$, and poor agreement was $0.01-0.40$. All the above listed analyses were performed using IBM SPSS Statistics 22 (IBM Corp.). A P value of $<0.05$ was considered statistically significant. Scatter plots were generated using Origin 2019 (OriginLab Corp.).

\section{Results}

\section{Phantom study}

A total of 429 image series were successfully reconstructed for the phantom study. The average SNR and CNR increased with the tube current (from 20 to $80 \mathrm{mAs}$ ). Higher SNR and CNR were observed in the iDose ${ }^{4}$ and IMR groups compared to the FBP group (all $P<0.05$ ). Among them, IMR level 3 had the highest value (Figure 2). For the iDose ${ }^{4}$ group and IMR groups, the average SNR and CNR increased as the iterative level increased.

For some tube current settings, the SNR and CNR averages for iDose ${ }^{4}$ level 7 were significantly higher than those for IMR level 1, while no significant differences were found for other tube current settings (Table 1).

The total AS from the reference image series $(120 \mathrm{kVp}$, $80 \mathrm{mAs}, \mathrm{FBP})$ was $680.95 \pm 3.29$. Compared with the reference image series, no significant differences (all $\mathrm{P}>0.05$ ) in AS were observed from the image series reconstructed with FBP and iDose ${ }^{4}$ (level 1-7), while the AS from the IMR (level 1-3) image series was significantly lower (all $\mathrm{P}<0.05$ ). For the insert with a HA density of $800 \mathrm{mg} / \mathrm{cm}^{3}$, all of the ASs from the IMR group were significantly lower 
Table 1 Comparison of SNR and CNR between the iDose ${ }^{4}$ level 7 group and the IMR level 1 group $(\bar{x} \pm$ s)

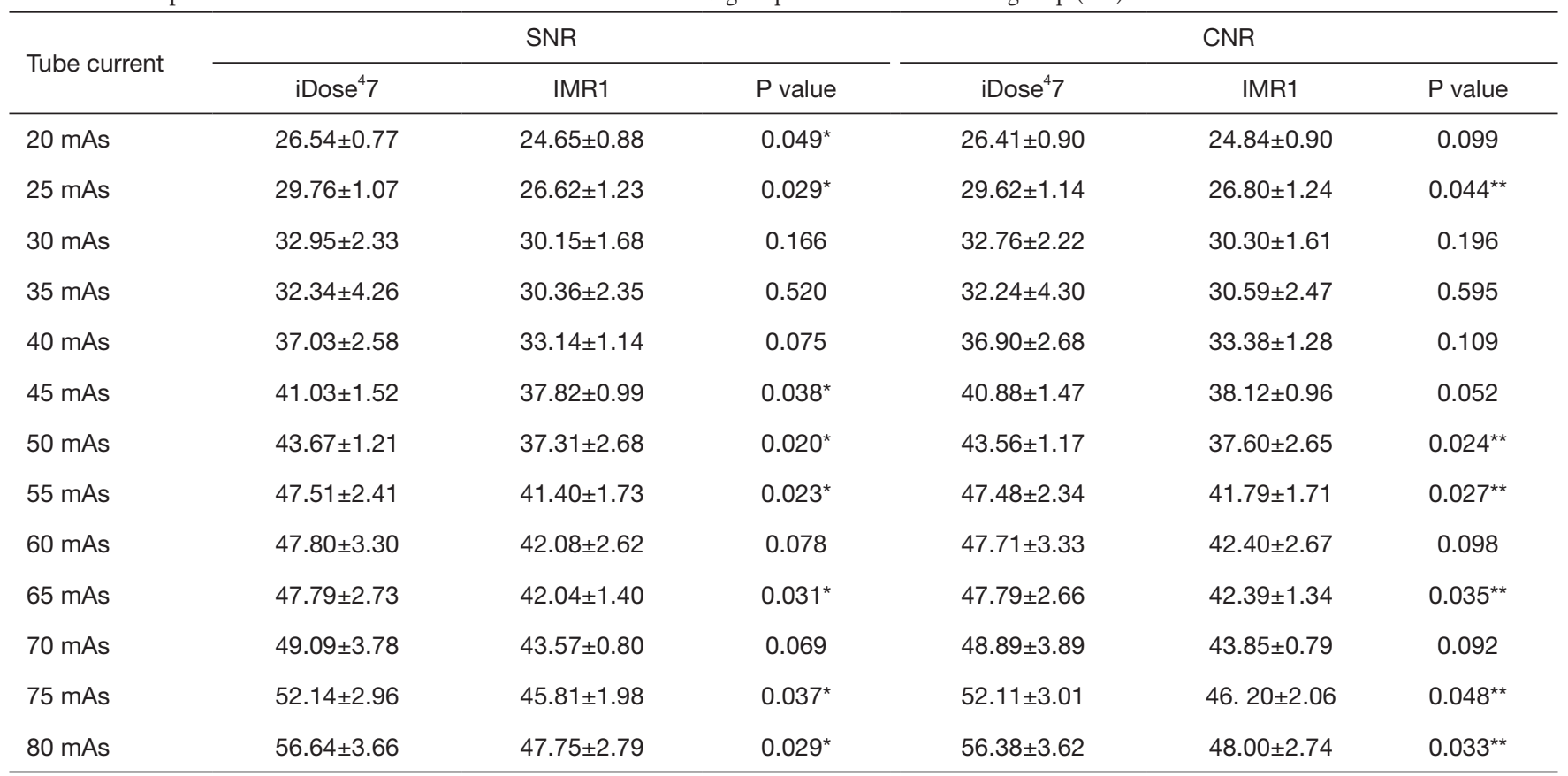

*, SNR from iDose 4 groups were significantly higher than that from IMR1 groups; ${ }^{\star \star}$, CNR from iDose ${ }^{4} 7$ groups were significantly higher than that from IMR1 groups. SNR, signal-to-noise ratio; CNR, contrast-to-noise ratio; IMR, iterative model reconstruction; iDose ${ }^{4} 7$, iDose ${ }^{4}$ level 7; IMR1, IMR level 1.

than those from the FBP group. For the insert with a HA density of $400 \mathrm{mg} / \mathrm{cm}^{3}$, there was no significant difference in AS between the IMR and FBP groups at the tube current settings of 35 and $50 \mathrm{mAs}$, while the AS from the IMR group was significantly lower than that from the FBP group for the other tube current settings. For the insert with a HA density of $200 \mathrm{mg} / \mathrm{cm}^{3}$, the AS from the IMR group was significantly lower than that from the FBP group at the tube current settings of 20 and $25 \mathrm{mAs}$, but significantly higher at the tube current settings from $55 \mathrm{mAs}$ to $80 \mathrm{mAs}$, with no significant differences being observed at the other tube current settings. The detailed results are listed in Tables 2,3,4,5. For the IMR group, in comparison with the standard radiation dose image series $(80 \mathrm{mAs})$, the total AS from the ultra-low radiation dose image series (20-40 mAs) was significantly lower (all $\mathrm{P}<0.05$ ), while the other low radiation dose image series showed no significant difference (Table 6). Therefore, the tube current of $50 \mathrm{mAs}$ was selected for the clinical study.

\section{Correction factor}

The correction factor was derived from regression analysis between the FBP and IMR level 2 image series using the
RRD protocol (120 kV, $50 \mathrm{mAs})$. The correction factor for the RRD protocol was $1.14\left(\mathrm{R}^{2}=0.99, \mathrm{P}<0.05\right.$, Figure 3$)$.

\section{Clinical study}

A total of 40 patients underwent CAC scanning. Three image series (FBP, iDose level 4, and IMR level 2) were reconstructed for each acquisition, and no motion artifacts were detected. The patient characteristics are summarized in Table 7.

The median AS from the iDose ${ }^{4}$ and IMR groups were both significantly lower than those from the FBP image series [112.89 (63.01, 314.09), 113.22 (64.78, 364.95) vs. 118.59 (65.05, 374.48), both $\mathrm{P}<0.05]$. After applying the correction factor, the adjusted AS from the IMR image series group showed no significant difference with the FBP group [126.48 (69.62, 355.85) vs. 118.59 (65.05, 374.48), $\mathrm{P}=0.145]$ (Figure 4).

The AS-based risk stratification for different groups are shown in Table 8. Using the corrected AS, 1 patient was reclassified from $1-100$ to $101-400$, and 2 patients were reclassified from 101-400 to $>400$. The agreement in risk stratification between FBP and IMR improved from 0.81 to 0.85 .

For the clinical study using the RRD CAC protocol, the 


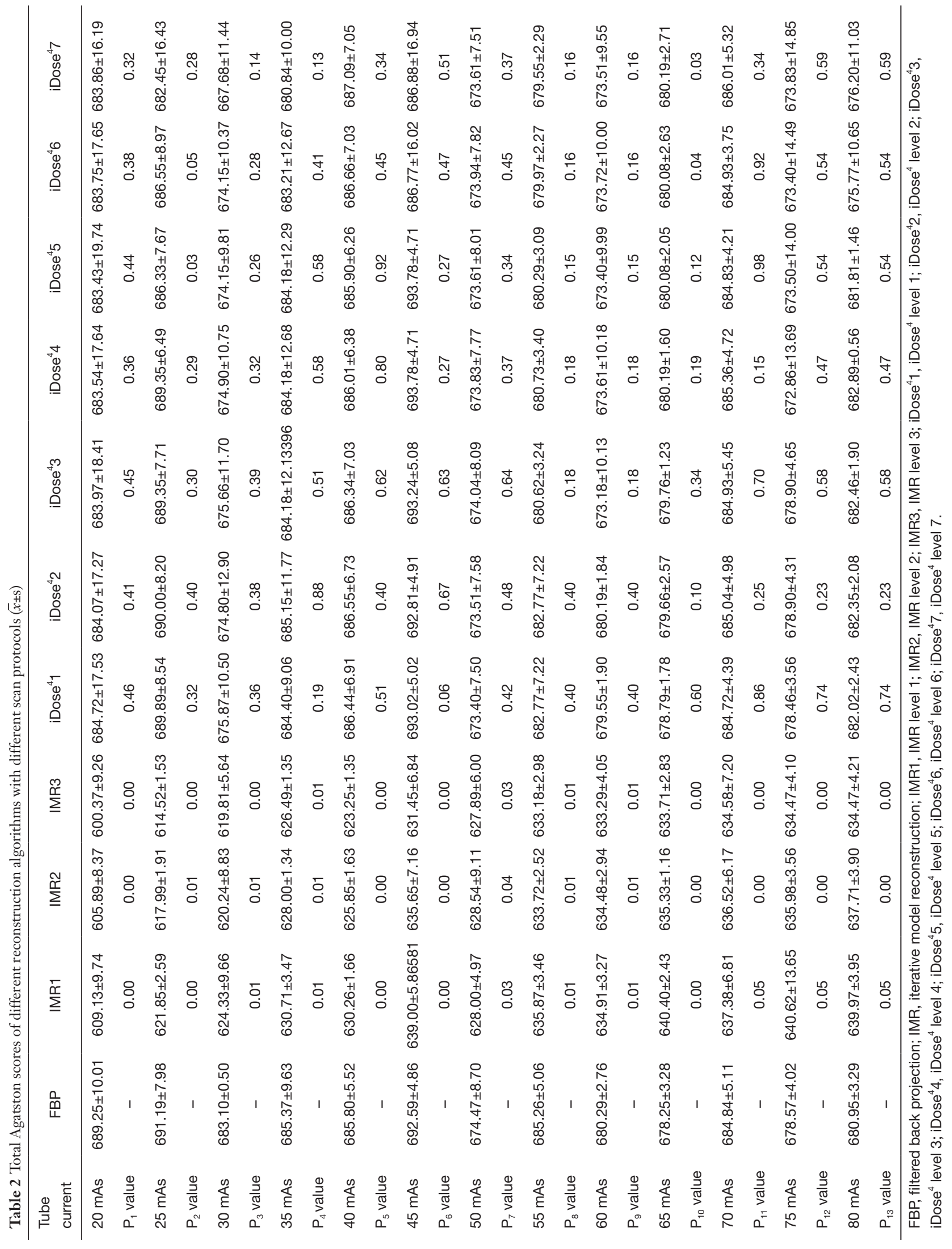




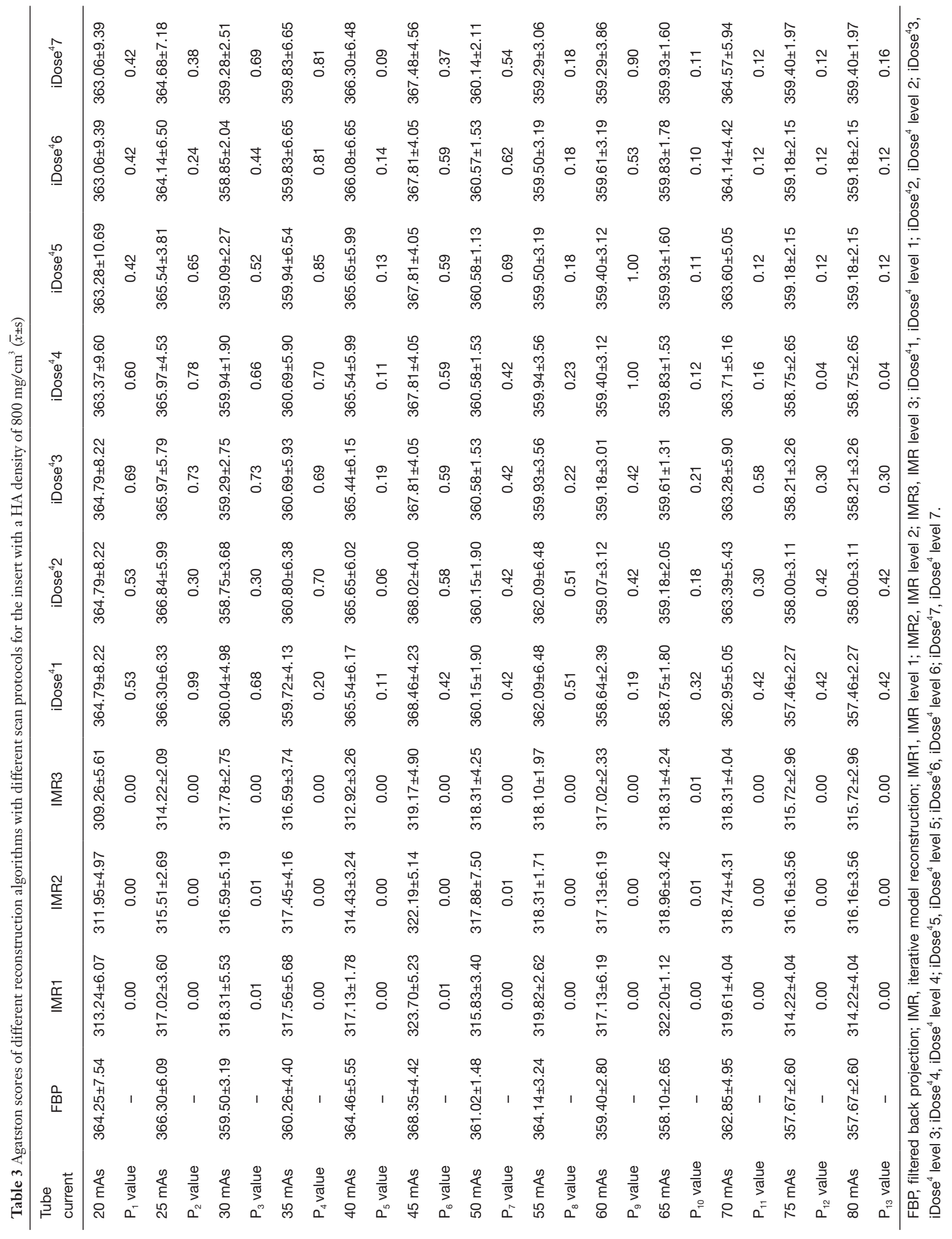




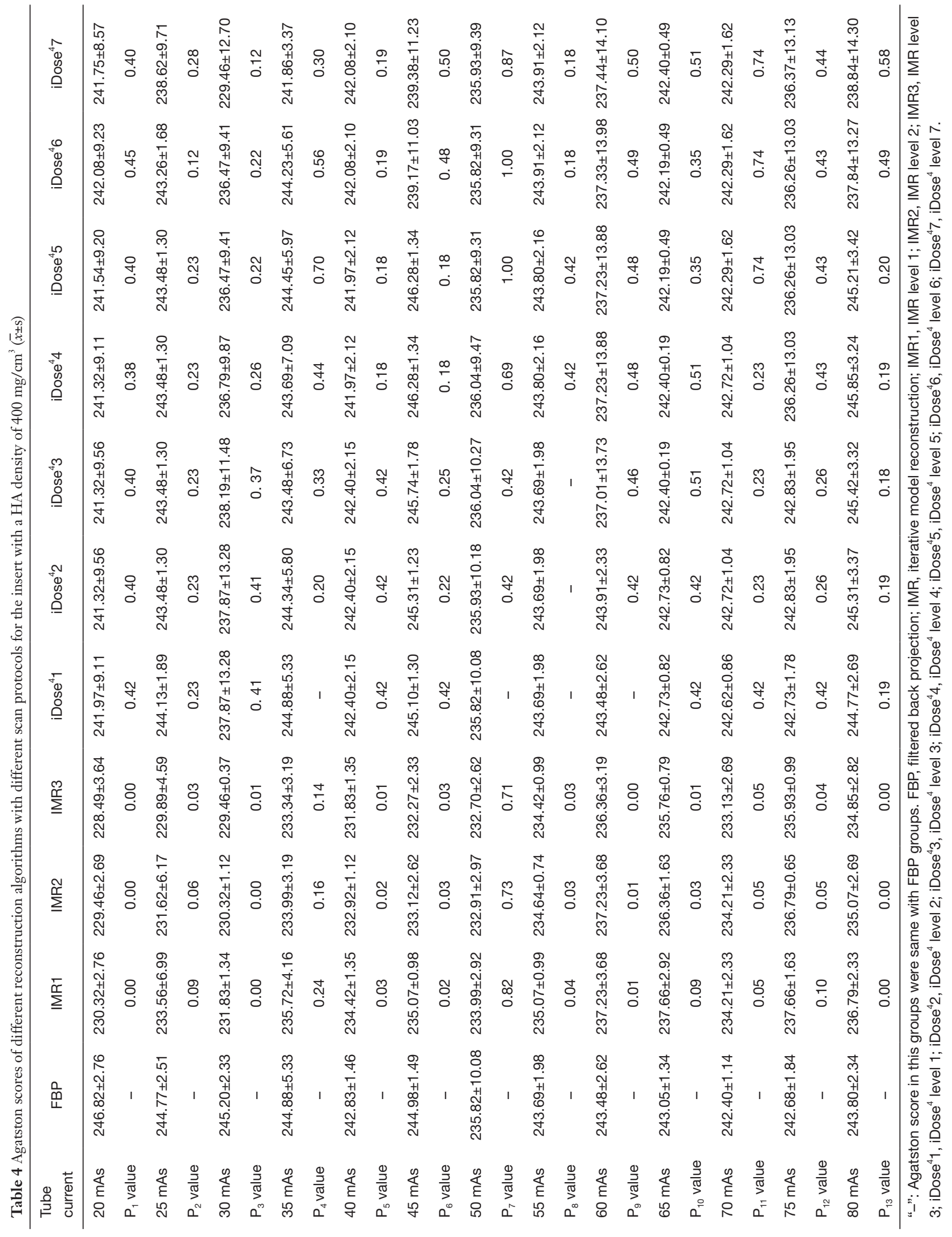




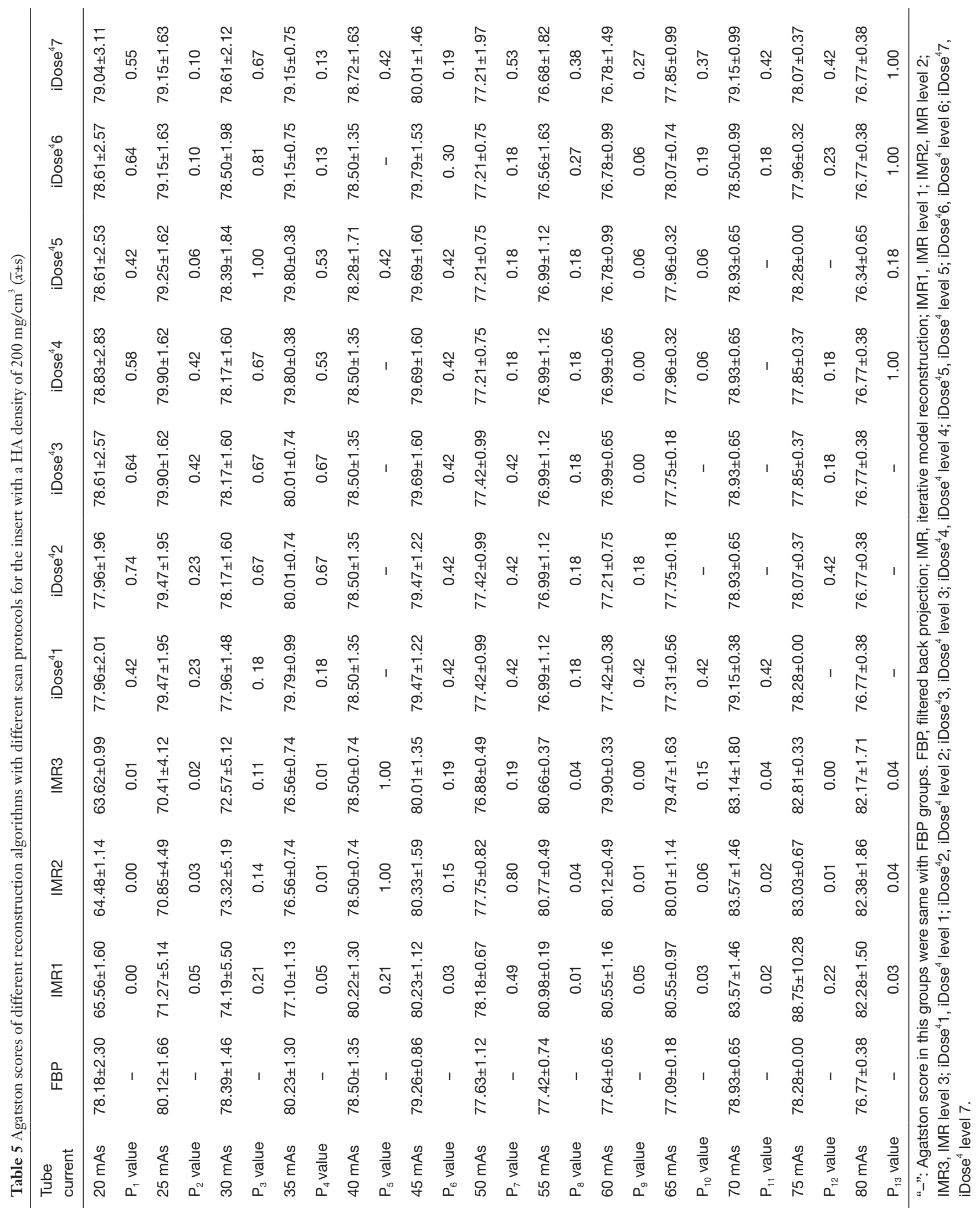




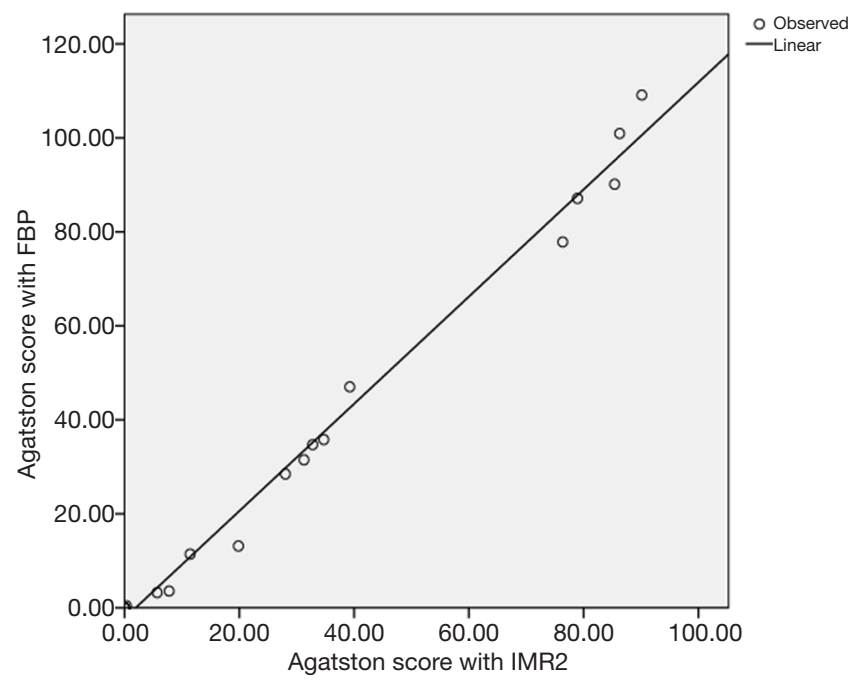

Figure 3 Linear regression equation of Agatston scores between FBP and IMR2 with the RRD protocol. FBP, filtered back projection; IMR, iterative model reconstruction; IMR2, IMR level 2; RRD, reduced radiation dose.

Table 7 Patients characteristics [median (quartile)] ( $\mathrm{n}=40)$

\begin{tabular}{lc}
\hline Characteristics & Variable \\
\hline Male & 19 \\
Female & 21 \\
Age (year) & $62(53,68)$ \\
Heart rate (bpm) & $70(66,79)$ \\
\hline
\end{tabular}

Table 8 Agatston score-based risk stratification for the different groups using different scan protocols $(\mathrm{n}=40)$

\begin{tabular}{lcccc}
\hline AS & FBP & iDose $^{4} 4$ & IMR2 & IMR2 after correction \\
\hline 0 & 0 & 0 & 0 & 0 \\
$1-100$ & 13 & 14 & 14 & 13 \\
$101-400$ & 14 & 13 & 17 & 16 \\
$>400$ & 13 & 13 & 9 & 11 \\
\hline
\end{tabular}

AS, Agatston score; FBP filtered back projection; IMR, iterative model reconstruction; iDose 4 , iDose ${ }^{4}$ level 4; IMR2, IMR level 2. 


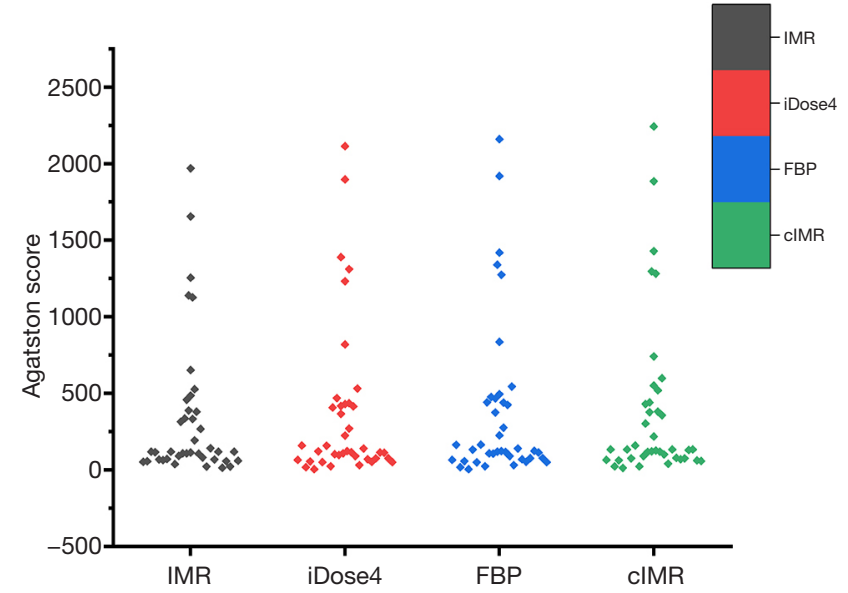

Figure 4 Agatston scores for the patients using different reconstruction algorithms and corrected Agatston scores for the IMR group. IMR, iterative model reconstruction; FBP, filtered back projection; cIMR, IMR after correction.

mean CTDIvol was $4.9 \mathrm{mGy}$, DLP was $41.9 \mathrm{mGy} \times \mathrm{cm}$, and $\mathrm{ED}$ was $0.69 \mathrm{mSv}$.

\section{Discussion}

Our data suggested that the IMR (level 2) algorithm could be applied to the CAC RRD scanning protocol with lower image noise. After applying the correction factor, we found no difference in AS between the IMR group and the FBP group. Nevertheless, the risk stratification of the patients was underestimated even when using the corrected AS. Moreover, the phantom study results implied that the AS was significantly lower when using an ultra-low radiation dose (20-40 mAs) scan compared to a normal radiation dose (80 mAs) scan using the IMR algorithm.

Willemink et al. (20) and den Harder et al. (21) performed a similar study by examining the maximal radiation dose reduction for the CAC scan using different IR algorithms and evaluating the AS results. They reported that $20 \mathrm{mAs}$ could be applied for a CAC scan with a radiation dose reduction of $60-80 \%$ if the highest IR level was applied, and the reclassification rates were maintained below $15 \%$. Some studies have also reported that the IR algorithm could reduce image noise and blooming artifacts using an ultra-low radiation dose scan protocol. They provided equivalent CNRs with an FBP reconstruction algorithm using a normal radiation dose scan protocol $(16,22)$. However, other studies have reported a negative correlation between AS and IR levels $(15,16,22,23)$, which can affect patient risk stratification. Our phantom study suggested that the IMR algorithm could significantly decrease the image noise, resulting in higher CNR, and underestimate AS and risk stratification compared with the FBP algorithm, which was consistent with previous studies. Additionally, for the IMR image series, no difference in AS was observed when applying tube current settings from 75 to $50 \mathrm{mAs}$ compared with the $80 \mathrm{mAs}$ setting. However, AS was significantly lower when using a setting from $40 \mathrm{mAs}$ to $20 \mathrm{mAs}$ compared with the $80 \mathrm{mAs}$ setting. Based on the phantom study data, $50 \mathrm{mAs}$ was selected for the RRD protocol tube current setting in our clinical study.

The AS is the most widely used method to quantify calcification of the coronary artery (6). However, the AS method cannot be used to evaluate information on the number or size of calcified coronary lesions or the regional distribution of calcification within the coronary tree. Furthermore, the weighting factor of AS is strongly affected by acquisition and reconstruction parameters, such as tube voltage and current, reconstruction algorithms, or image slice thickness (7). Although several new methods (like calcium volume score, calcium mass score) have been proposed to quantify calcification of the coronary artery, only AS has been validated by a large scale clinical trial (24), after which the guidelines with an evidence level of IIb for the assessment of asymptomatic adults at intermediate risk were written (4). Thus, another strategy is to optimize the acquisition parameters so as to improve image quality, decrease radiation dose, and reduce the variability of AS accordingly. Many studies have reported that IR algorithms can reduce radiation dose and improve image quality. However, they can also cause restratification (21-23,25-28). den Harder et al. (21) found that risk category of 21-25\% and $18 \%$ patients were reclassified with IMR and HIR respectively at RRD levels. Moreover, Obmann et al. (23) reported that the risk stratification of $5.4 \%$ in patients was different for the HIR group with the highest iteration level compared to the FBP group. Caruso et al. (28) compared risk stratifications in the advanced modeled IR (ADMIRE) groups and the FBP group. In their study, 6 patients (15\%) were reclassified into a lower risk category at ADMIRE level 3, while 15 patients $(37.5 \%)$ were reclassified into a lower risk category at ADMIRE level 5. In our study, 1 patient was reclassified into the lower risk category with iDose ${ }^{4}$ level 4 , and 5 patients were reclassified into the lower risk category with IMR level 2. After applying the correction factor for the IMR group, 2 patients were 
reclassified into the lower risk category, and the agreement in risk stratification between FBP and IMR increased from 0.81 to 0.85 . Fan et al. (18) suggested modifying risk stratification thresholds based on the best diagnostic cutoff value obtained from the receiver operating characteristic (ROC) curves for reducing radiation doses in scan protocols using the IMR algorithm. The new CAC scoring risk stratification defined values of: $1-87$ as low risk, $88-255$ as moderate risk, and $>255$ as high risk. However, these modified thresholds have not been widely validated, and are scanner-dependent.

This study has a few limitations. Firstly, the phantom was scanned 3 times with each scan protocol, which might have introduced data bias. However, the data were stable considering the standardized physical characteristics of the phantom. Secondly, our phantom study did not simulate different somatotypes. Thirdly, all patients were scanned with the RRD protocol as suggested, but were not all scanned with the reference protocol due to ethical considerations, as there was higher radiation dose used with the reference protocol. Fourthly, to test the effect of different IR algorithms on the assessment of CAC, more severely calcified plaques or high CAC cases are needed to determine its effect on the quantification of CAC. Finally, large-scale patient studies should be performed to further validate the proposed method.

\section{Conclusions}

In conclusion, this study demonstrates that the RRD CAC scoring scan using the IMR reconstruction algorithm is clinically feasible, and a correction factor can help reduce AS underestimation effects.

\section{Acknowledgments}

Funding: None.

\section{Footnote}

Conflicts of Interest: All authors have completed the ICMJE uniform disclosure form (available at http://dx.doi. org/10.21037/qims-20-437). The authors have no conflicts of interest to declare.

Ethical Statement: The Ethics Committee of Central China Fuwai Hospital approved this study. Informed consent: Written informed consent was obtained from the patients for publication of this study and any accompanying images.

Open Access Statement: This is an Open Access article distributed in accordance with the Creative Commons Attribution-NonCommercial-NoDerivs 4.0 International License (CC BY-NC-ND 4.0), which permits the noncommercial replication and distribution of the article with the strict proviso that no changes or edits are made and the original work is properly cited (including links to both the formal publication through the relevant DOI and the license). See: https://creativecommons.org/licenses/by-nc-nd/4.0/.

\section{References}

1. Pletcher MJ, Sibley CT, Pignone M, Vittinghoff E, Greenland P. Interpretation of the coronary artery calcium score in combination with conventional cardiovascular risk factors: the Multi-Ethnic Study of Atherosclerosis (MESA). Circulation 2013;128:1076-84.

2. Budoff MJ, Mayrhofer T, Ferencik M, Bittner D, Lee KL, Lu MT, Coles A, Jang J, Krishnam M, Douglas PS, Hoffmann U, Investigators P. Prognostic value of coronary artery calcium in the promise study (prospective multicenter imaging study for evaluation of chest pain). Circulation 2017;136:1993-2005.

3. Mahabadi AA, Möhlenkamp S, Lehmann N, Kälsch H, Dykun I, Pundt N, Moebus S, Jöckel KH, Erbel R. CAC score improves coronary and cv risk assessment above statin indication by esc and aha/acc primary prevention guidelines. JACC Cardiovascular Imaging 2017;10:143-53.

4. Greenland P, Alpert JS, Beller GA, Benjamin EJ, Budoff MJ, Fayad ZA, Foster E, Hlatky MA, Hodgson JM, Kushner FG, Lauer MS, Shaw LJ, Smith SC Jr, Taylor AJ, Weintraub WS, Wenger NK, Jacobs AK, Smith SC Jr, Anderson JL, Albert N, Buller CE, Creager MA, Ettinger SM, Guyton RA, Halperin JL, Hochman JS, Kushner FG, Nishimura R, Ohman EM, Page RL, Stevenson WG, Tarkington LG, Yancy CW; American College of Cardiology Foundation; American Heart Association. 2010 ACCF/AHA guideline for assessment of cardiovascular risk in asymptomatic adults: a report of the American College of Cardiology Foundation/American Heart Association Task Force on Practice Guidelines. J Am Coll Cardiol 2010;56:e50-103.

5. Liu T, Yuan X, Wang C, Sun M, Jin S, Dai X. Quantification of plaque characteristics detected by dual source computed tomography angiography to predict myocardial ischemia as assessed by single photon emission 
computed tomography myocardial perfusion imaging. Quant Imaging Med Surg 2019;9:711-21.

6. Agatston AS, Janowitz WR, Hildner FJ, Zusmer NR, Viamonte M, Detrano R. Quantification of coronary artery calcium using ultrafast computed tomography. J Am Coll Cardiol 1990;15:827-32.

7. Blaha MJ, Mortensen MB, Kianoush S, Tota-Maharaj R, Cainzos-Achirica $M$. Coronary artery calcium scoring: is it time for a change in methodology? JACC Cardiovasc Imaging 2017;10:923-37.

8. Huang X, Gao S, Ma Y, Lu X, Jia Z, Hou Y. The optimal monoenergetic spectral image level of coronary computed tomography (CT) angiography on a dual-layer spectral detector CT with half-dose contrast media. Quant Imaging Med Surg 2020;10:592-603.

9. Vonder M, van der Werf NR, Leiner T, Greuter MJW, Fleischmann D, Vliegenthart R, Oudkerk M, Willemink MJ. The impact of dose reduction on the quantification of coronary artery calcifications and risk categorization: A systematic review. J Cardiovasc Comput Tomogr 2018;12:352-63.

10. Gassenmaier T, Allmendinger T, Kunz AS, VeyhlWichmann M, Ergun S, Bley TA, Petritsch B. In vitro evaluation of a new iterative reconstruction algorithm for dose reduction in coronary artery calcium scoring. Acta Radiol Open 2017;6:2058460117710682.

11. Vonder M, Pelgrim GJ, Meyer M, Henzler T, Oudkerk M, Vliegenthart R. Dose reduction techniques in coronary calcium scoring: The effect of iterative reconstruction combined with low tube voltage on calcium scores in a thoracic phantom. Eur J Radiol 2017;93:229-35.

12. van Osch JA, Mouden M, van Dalen JA, Timmer JR, Reiffers S, Knollema S, Greuter MJ, Ottervanger JP, Jager PL. Influence of iterative image reconstruction on CT-based calcium score measurements. Int J Cardiovasc Imaging 2014;30:961-7.

13. Károlyi M, Szilveszter B, Kolossváry M, Takx RA, Celeng C, Bartykowszki A, Jermendy ÁL, Panajotu A, Karády J, Raaijmakers R, Giepmans W, Merkely B, MaurovichHorvat P. Iterative model reconstruction reduces calcified plaque volume in coronary CT angiography. Eur J Radiol 2017;87:83-9.

14. den Harder AM, Willemink MJ, Bleys RL, de Jong PA, Budde RP, Schilham AM, Leiner T. Dose reduction for coronary calcium scoring with hybrid and model-based iterative reconstruction: an ex vivo study. Int J Cardiovasc Imaging 2014;30:1125-33.

15. Oda S, Utsunomiya D, Nakaura T, Funama Y, Yuki H,
Kidoh M, Hirata K, Taguchi N, Honda K, Takaoka H, Iyama Y, Katahira K, Noda K, Oshima S, Tokuyasu S, Yamashita Y. The influence of iterative reconstruction on coronary artery calcium scoring-phantom and clinical studies. Acad Radiol 2017;24:295-301.

16. Szilveszter B, Elzomor H, Karolyi M, Kolossvary M, Raaijmakers R, Benke K, Celeng C, Bartykowszki A, Bagyura Z, Lux A, Merkely B, Maurovich-Horvat P. The effect of iterative model reconstruction on coronary artery calcium quantification. Int J Cardiovasc Imaging 2016;32:153-60.

17. McCollough CH, Ulzheimer S, Halliburton SS, Shanneik K, White RD, Kalender WA. Coronary artery calcium: a multi-institutional, multimanufacturer international standard for quantification at cardiac CT. Radiology 2007;243:527-38.

18. Fan R, Shi X, Qian Y, Wang Y, Fan L, Chen R, Xiao Y, Liu S. Optimized categorization algorithm of coronary artery calcification score on non-gated chest low-dose CT screening using iterative model reconstruction technique. Clin Imaging 2018;52:287-91.

19. McCollough C, Edyvean S, Cody D, Geise R, Gould B. AAPM report no. 96: the measurement, reporting, and management of radiation dose in CT-report of AAPM Task Group 23 of the Diagnostic Imaging Council CT Committee. American Association of Physicists in Medicine. Available online: http://www.aapm.Org/pubs/ reports/RPT96

20. Willemink MJ, den Harder AM, Foppen W, Schilham AMR, Rienks R, Laufer EM, Nieman K, de Jong PA, Budde RPJ, Nathoe HM, Leiner T. Finding the optimal dose reduction and iterative reconstruction level for coronary calcium scoring. J Cardiovasc Comput Tomogr 2016;10:69-75.

21. den Harder AM, Wolterink JM, Willemink MJ, Schilham AMR, de Jong PA, Budde RPJ, Nathoe HM, Išgum I, Leiner T. Submillisievert coronary calcium quantification using model-based iterative reconstruction: A withinpatient analysis. Eur J Radiol 2016;85:2152-9.

22. van der Werf NR, Willemink MJ, Willems TP, Greuter MJW, Leiner T. Influence of iterative reconstruction on coronary calcium scores at multiple heart rates: a multivendor phantom study on state-of-the-art CT systems. Int J Cardiovasc Imaging 2018;34:947-57.

23. Obmann VC, Klink T, Heverhagen JT, Stork A, Laqmani A, Adam G, Begemann PG. Impact of hybrid iterative reconstruction on Agatston coronary artery calcium scores in comparison to filtered back projection in native cardiac 
CT. RöFo 2015;187:372-9.

24. Vonder M, van der Aalst CM, Vliegenthart R, van Ooijen PMA, Kuijpers D, Gratama JW, de Koning HJ, Oudkerk M. Coronary artery calcium imaging in the robinsca trial. Acad Radiol 2018;25:118-28.

25. Willemink MJ, Takx RA, de Jong PA, Budde RP, Bleys RL, Das M, Wildberger JE, Prokop M, Buls N, de Mey J, Schilham AM, Leiner T. The impact of CT radiation dose reduction and iterative reconstruction algorithms from four different vendors on coronary calcium scoring. Eur Radiol 2014;24:2201-12.

26. Sulaiman N, Soon J, Park JK, Naoum C, Kueh SH, Blanke P, Murphy D, Ellis J, Hague CJ, Leipsic J. Comparison of low-dose coronary artery calcium scoring using low tube

Cite this article as: Pan YK, Sun MH, Wang JJ, Chen XB, Kan XJ, Ge YH, Guo ZP. Effect of different reconstruction algorithms on coronary artery calcium scores using the reduced radiation dose protocol: a clinical and phantom study. Quant Imaging Med Surg 2021;11(4):1504-1517. doi: 10.21037/qims20-437 current technique and hybrid iterative reconstruction vs. filtered back projection. Clin Imaging 2017;43:19-23.

27. Tang YC, Liu YC, Hsu MY, Tsai HY, Chen CM. Adaptive iterative dose reduction $3 \mathrm{D}$ integrated with automatic tube current modulation for CT coronary artery calcium quantification: comparison to traditional filtered back projection in an anthropomorphic phantom and patients. Acad Radiol 2018;25:1010-7.

28. Caruso D, De Cecco CN, Schoepf UJ, Felmly LM, VargaSzemes A, Mangold S, Canstein C, Allmendinger T, Fuller SR, Laghi A, Wichmann JL. Correction factors for CT coronary artery calcium scoring using advanced modeled iterative reconstruction instead of filtered back projection. Acad Radiol 2016;23:1480-9. 